\title{
Total and free fatty acids content during the ripening of artisan and industrially manufactured "Chorizo de cebolla"
}

\author{
By Inmaculada Franco, Amalia Martínez, Bernardo Prieto and Javier Carballo* \\ Área de Tecnología de los Alimentos. Facultad de Ciencias de Orense. Universidad \\ de Vigo. 32004 Orense, Spain. E-mail: carbatec@uvigo.es
}

\section{RESUMEN}

Efecto del tipo de elaboración (artesanal o industrial) sobre el contenido en ácidos grasos totales y libres a lo largo de la maduración del "Chorizo de cebolla".

El chorizo de cebolla es un embutido tradicional elaborado en el noroeste de España. En cuatro partidas fabricadas por procedimientos artesanales y 4 elaboradas industrialmente se determinó a lo largo de la maduración los contenidos en ácidos grasos totales y libres, tomando en cada partida muestras de masa antes de embutir (0 días) y de chorizo a los 2, 7, 14, 21, 28 y 42 días de maduración. El perfil de ácidos grasos totales de los dos tipos de embutidos coincide básicamente con el encontrado por otros autores en grasa de cerdo. Sin embargo, ambos tipos de chorizo (artesanal e industrial) difirieron significativamente $(p<0.05)$ en el porcentaje de ácidos grasos totales saturados e insaturados. Los chorizos elaborados artesanalmente presentaron porcentajes de ácidos grasos saturados significativamente superiores a los encontrados en los chorizos industriales.

El contenido en ácidos grasos libres totales experimentó a lo largo de la maduración un incremento significativo $(p<0.05)$ desde valores medios de $459 \pm 243 \mathrm{mg} / 100 \mathrm{~g}$ de grasa en la masa hasta $3687 \pm 1670 \mathrm{mg} / 100 \mathrm{~g}$ de grasa en chorizo de 42 días, en los chorizos artesanales, y desde $560 \pm 317 \mathrm{mg} / 100 \mathrm{~g}$ de grasa hasta $5157 \pm 3673 \mathrm{mg} / 100 \mathrm{~g}$ en los industriales. Debido a la gran variabilidad entre las diferentes partidas, no se encontraron diferencias estadísticamente significativas en los contenidos en ácidos grasos libres totales entre ambos tipos de chorizo, aunque al final de la maduración el contenido medio fue 1.4 veces superior en los chorizos industriales. En ambos tipos de chorizo, los ácidos grasos libres mayoritarios al final de la maduración fueron, por este orden, el oleico, linoleico y palmítico, cuya suma representó en ambos casos el $83 \%$ de los ácidos grasos libres totales.

PALABRAS-CLAVE: Ácidos grasos libres - Ácidos grasos totales - Chorizo de cebolla - Embutidos crudos-curados - Lipólisis - Maduración.

\section{SUMMARY}

Total and free fatty acids content during the ripening of artisan and industrially manufactured "Chorizo de cebolla".

"Chorizo de cebolla" is a traditional sausage made in the north west of Spain. In four batches manufactured by artisanal methods and 4 manufactured by industrial ones the contents of total and free fatty acids were assessed throughout ripening, taking from every batch samples of the mass before stuffing ( 0 days) and of the sausage after $2,7,14,21,28$ and 42 days of ripening. The profile of total fatty acids of the two types of sausage basically coincide with that found by other authors in pork fat. However, both types of sausage (artisanal and industrial) differ significantly $(p<$ 0.05 ) in the percentage of totally saturated and unsaturated fatty acids. The artisanal manufactured sausages showed percentages of saturated fatty acids significantly superior to those found in industrial sausages.

The content of total free fatty acids underwent throughout ripening a significant increase $(p<0.05)$ from average values of $459 \pm 243 \mathrm{mg} / 100 \mathrm{~g}$ of fat in the mass up to $3687 \pm 1670 \mathrm{mg} / 100$ $\mathrm{g}$ of fat in the sausage of 42 days in the artisanal sausage, and from $560 \pm 317 \mathrm{mg} / 100 \mathrm{~g}$ of fat up to $5157 \pm 3673 \mathrm{mg} / 100 \mathrm{~g}$ in the industrial ones. Owing to the great variation between the different batches, significant differences were not found in the contents of total free fatty acids between both types of sausages, though at the end of ripening the average content was 1.4 times superior in the industrial sausages. In both types of sausages, the main free fatty acids at the end of ripening were, in this order, oleic, linoleic and palmitic, whose addition represented $83 \%$ of total free fatty acids.

KEY-WORDS: Chorizo de cebolla - Free fatty acids - Lipolysis - Raw-cured sausages - Ripening - Total fatty acids.

\section{INTRODUCTION}

Salting and dehydration processes are presumably the oldest ways of preserving foods. Probably men initially started drying large pieces of meat in the open air and later verified that the use of salt, added to dehydration, increased the usable life of the meat pieces and even improved their aroma. Later they substantiated that cutting the meat into smaller pieces, salting them and putting them in animal tripe remarkably increased their preservability and improved their organoleptic characteristics. Lore about this type of meat products is very ancient, a type of sausage was already manufactured in China 2500 years ago (Leistner and Dressel, 1986), and sausages were consumed in abundance by Babylonians, Greeks and Romans (Pederson, 1971).

This preserving technology has been maintained and diversified. It exist a great number of national and regional varieties of sausages nowadays.

Spain is in the fourth place as far as the production of meat products is concerned in the European Union, coming after Germany, Italy and France. Of the 917 915 tons of meat products manufacture in Spain in 1999, 157000 corresponded to raw-cured sausages (MAPA, 2000).

The final characteristics of raw-cured sausages as far as the appearance, taste and aroma are concerned, the same as to their preservability and 
safety, are a consequence of a complex united of physico-chemical, biochemical and microbiological modifications that take place during the fermentation-ripening. Without detracting from the importance of dehydration, the changes in physico-chemical parameters such as $\mathrm{pH}$ or $\mathrm{Aw}$, and the degradative phenomena of proteins, the degradation of lipids is probably the biochemical phenomenon most relevant during ripening of sausages, because their influence on the organoleptic characteristics of the final product (Cantoni et al., 1966; Demeyer et al., 1974). Lipids undergo a series of transformations during sausage ripening which include hydrolytic processes, releasing of fatty acids (those of short chain intervene in a direct way in the development of the aroma), and oxidation of these fatty acids with the formation of peroxides and volatile compounds which contribute to the aroma of the final product. Lipolysis in sausages starts during fermentation (Nagy et al., 1989; Roncalés et al., 1991; Santamaría et al., 1992) and progresses in a more or less uniform way during all the ripening stage (Langner, 1969; Johansson et al., 1994).

"Chorizo de cebolla" is a raw-cured sausage which is manufactured, as much in an artisanal way as an industrial one, in Galicia (NW of Spain). It is included in the "Spanish Catalogue of sausages and cured hams" (MAPA, 1983) and has reached a really important volumes of production which has been increasing all the time during the last years. Studies carried out until now on this sausage only concern the microbiological changes that take place during the manufacture (García Fontán et al., 1998) and the determination of the indexes of the characterization of the fat during the manufacture (Salgado et al., 1999) and in the end product (Rodríguez et al., 2001). At present, data do not exist on the rest of biochemical changes which take place throughout the manufacturing process.

The aim of this article is the study of the contents in total and free fatty acids throughout the manufacture of "Chorizo de cebolla", as well as to establish the

Table I

Chromatographic conditions used in the identification and quantification of the fatty acids

\begin{tabular}{ccccc}
\hline $\begin{array}{c}\text { Temperature } \\
\text { ramps }\end{array}$ & ${ }^{\circ} \mathrm{C}$ /minute & $\begin{array}{c}\text { Temperature } \\
\left({ }^{\circ} \mathrm{C}\right)\end{array}$ & $\begin{array}{c}\text { Holding time } \\
\text { (minutes) }\end{array}$ & $\begin{array}{c}\text { Passed time } \\
\text { (minutes) }\end{array}$ \\
\hline Initial & & 50 & 1.00 & 1.00 \\
Ramp 1 & 10 & 150 & 1.00 & 12.00 \\
Ramp 2 & 12 & 180 & 0.00 & 14.50 \\
Ramp 3 & 2 & 188 & 6.00 & 24.50 \\
Ramp 4 & 2 & 220 & 2.00 & 42.50 \\
Ramp 5 & 20 & 260 & 0.00 & 44.50 \\
\hline
\end{tabular}

incidence which the type of manufacture (artisanal or industrial) has on these contents. This work is included in a broader research project whose object is the study of the biochemical and microbiological modifications which take place during manufacture of this sausage.

\section{MATERIALS AND METHODS}

\subsection{Manufacture of the sausages and taking of samples}

To carry out this study eight batches of "Chorizo de cebolla" were made, four by artisanal means in private houses and the other four in pork meat industries.

In the industrial batches, the ingredients used in the composition of the mass (approximately per 150 $\mathrm{kg}$ ) were on average: dewlap, $70 \mathrm{~kg}$; bacon, $40 \mathrm{~kg}$; sweet paprika, $2.5 \mathrm{~kg}$; spicy paprika, $0.5 \mathrm{~kg}$; salt, $3 \mathrm{~kg}$; garlic, $1.5 \mathrm{~kg}$; marjoram, $0.075 \mathrm{~kg}$; onion (cebolla), 30 $\mathrm{kg}$; supplement BELL $\mathrm{n}^{\circ} 2 \mathrm{C}$ from Laboratorios $\mathrm{La}$ Campana (Chirivella, Valencia, Spain), $2.25 \mathrm{~kg}$. The supplement contained dehydrated skim milk, sugars, disodium diphosphate, soya proteins, salt, dextrin, sodium nitrite, potassium nitrate, sodium citrate, and red cochineal as colorant.

In the artisanal batches, the ingredients used (also approximately per $150 \mathrm{~kg}$ of mass) were on average: dewlap, $50 \mathrm{~kg}$; bacon, $20 \mathrm{~kg}$; cooked skin, $25 \mathrm{~kg}$; lung, $17 \mathrm{~kg}$; sweet paprika, $2.5 \mathrm{~kg}$; spicy paprika, $0.5 \mathrm{~kg}$; salt, $3 \mathrm{~kg}$; garlic, $1.5 \mathrm{~kg}$; marjoram, $0.1 \mathrm{~kg}$; onion, $10 \mathrm{~kg}$; pumpkin, $20 \mathrm{~kg}$.

Meat was triturated, minced with spices and additives and was left to stand during for approximately 24 hours refrigerated (around $5^{\circ} \mathrm{C}$ ). After this period it was mixed with the onion and pumpkin, which were triturated apart, and the resulting mass was stuffed in pork rectum tripe.

After being stuffed, the sausages were smoked for 5 days with oak and birch wood. After this, in the industrial manufacture they were transfered to a drying room where they stayed for the rest of the ripening process at $11{ }^{\circ} \mathrm{C}$ and $70 \%$ of relative humidity. In the artisanal manufacture, after smoking, the sausages were transfered to cool and ventilated rooms, where they stayed for the rest of the ripening process.

From each of the batches, samples of the mass before stuffing ( 0 days) and from the sausages after $2,7,14,21,28$, and 42 days of ripening were taken.

The samples were transfered to the laboratory where, after taking off the tripe, they were minced and triturated until a uniform mass was obtained. To preserve them adequately, they were kept inside airtight containers at a temperature of $-80^{\circ} \mathrm{C}$ until the time for the analysis. 
Table II

Evolution of the content of the different fatty acids (expressed as \% of the total fatty acids) during the manufacture of "Chorizo de cebolla" made by artisanal methods (means \pm standard deviations of four batches)

\begin{tabular}{|c|c|c|c|c|c|c|c|}
\hline & \multicolumn{7}{|c|}{ Ripening time (days) } \\
\hline & 0 & 2 & 7 & 14 & 21 & 28 & 42 \\
\hline $\mathrm{C}_{10}$ & $0.11 \pm 0.01^{\mathrm{a}}$ & $0.11 \pm 0.01^{\mathrm{a}}$ & $0.11 \pm 0.01^{\mathrm{a}}$ & $0.10 \pm 0.01^{\mathrm{a}}$ & $0.10 \pm 0.01^{\mathrm{a}}$ & $0.12 \pm 0.01^{\mathrm{a}}$ & $0.10 \pm 0.07^{\mathrm{a}}$ \\
\hline $\mathrm{C}_{12}$ & $0.10 \pm 0.01^{\mathrm{a}}$ & $0.10 \pm 0.00^{\mathrm{a}}$ & $0.10 \pm 0.01^{\mathrm{a}}$ & $0.10 \pm 0.00^{\mathrm{a}}$ & $0.10 \pm 0.01^{\mathrm{a}}$ & $0.11 \pm 0.01^{\mathrm{a} *}$ & $0.10 \pm 0.01^{\mathrm{a}}$ \\
\hline $\mathrm{C}_{14}$ & $1.25 \pm 0.16^{\mathrm{a}}$ & $1.18 \pm 0.01^{\mathrm{a}}$ & $1.17 \pm 0.12^{\mathrm{a}}$ & $1.18 \pm 0.01^{\mathrm{a}}$ & $1.18 \pm 0.01^{\mathrm{a}}$ & $1.25 \pm 0.01^{\mathrm{a}}$ & $1.17 \pm 0.14^{\mathrm{a}}$ \\
\hline $\mathrm{C}_{16}$ & $23.37 \pm 0.60^{\mathrm{a}}$ & $22.85 \pm 0.64^{\mathrm{a}}$ & $22.51 \pm 0.68^{\mathrm{a}}$ & $22.80 \pm 0.96^{\mathrm{a}}$ & $22.79 \pm 0.87^{\mathrm{a}}$ & $23.28 \pm 0.75^{\mathrm{a}}$ & $22.20 \pm 0.78^{\mathrm{a}}$ \\
\hline $\mathrm{C}_{18}$ & $13.08 \pm 0.51^{\mathrm{a}}$ & 13.8 & 13.01 & $13.65 \pm 1.49^{a \star}$ & $13.66 \pm 1.55^{\mathrm{a} \star}$ & $14.20 \pm 1.08^{\mathrm{a} \star}$ & 13.0 \\
\hline $\mathrm{C}_{20}$ & $0.51 \pm 0.06^{a}$ & $0.50 \pm 0.04^{a}$ & $0.62 \pm 0.06^{a}$ & $0.52 \pm 0.06^{a}$ & $0.52 \pm 0.05^{\mathrm{a}}$ & $0.35 \pm 0.20^{a}$ & $0.39 \pm 0.27^{\mathrm{a}}$ \\
\hline$C_{16: 1}$ & $1.91 \pm 0.06^{\mathrm{a}}$ & $1.66 \pm 0.12^{\mathrm{a} *}$ & $1.76 \pm 0.39^{a *}$ & $1.70 \pm 0.15^{\mathrm{a} \star}$ & $1.72 \pm 0.20^{\mathrm{a}^{*}}$ & $1.70 \pm 0.19^{a \star}$ & $1.82 \pm 0.34^{a}$ \\
\hline$C_{18: 1}$ & $45.95 \pm 2.16^{a}$ & $44.10 \pm 2.08^{\mathrm{a}}$ & $46.20 \pm 8.44^{a}$ & $44.11 \pm 2.18^{\mathrm{a}}$ & $43.83 \pm 2.10^{\mathrm{a}}$ & $43.27 \pm 0.50^{\mathrm{a}}$ & $45.13 \pm 2.07^{\mathrm{a}}$ \\
\hline$C_{18: 2}$ & $13.23 \pm 1.66^{a}$ & $14.94 \pm 2.07^{\mathrm{a}}$ & $13.81 \pm 2.30^{\mathrm{a}}$ & $15.09 \pm 2.14^{\mathrm{a}}$ & $15.47 \pm 1.78^{\mathrm{a}}$ & $15.06 \pm 1.69^{a}$ & $15.41 \pm 0.94^{\mathrm{a}}$ \\
\hline $\mathrm{C}_{18.3}$ & $0.48 \pm 0.14^{a}$ & $0.63 \pm 0.18^{\mathrm{a}}$ & $0.69 \pm 0.21^{\mathrm{a}}$ & $0.70 \pm 0.20^{\mathrm{a}}$ & $0.60 \pm 0.15^{a}$ & $0.66 \pm 0.18^{a}$ & $0.64 \pm 0.38^{\mathrm{a}}$ \\
\hline$C_{20: 4}$ & $0.02 \pm 0.03^{\mathrm{a}}$ & $0.06 \pm 0.08^{\mathrm{a}}$ & $0.03 \pm 0.07^{\mathrm{a}}$ & $0.04 \pm 0.08^{\mathrm{a}}$ & $0.03 \pm 0.07^{\mathrm{a}}$ & - & - \\
\hline S & $38.42 \pm 0.82^{\mathrm{a}}$ & $38.60 \pm 1.39^{a \star}$ & $37.52 \pm 2.11^{\mathrm{a} *}$ & $38.32 \pm 2.47^{\mathrm{a} *}$ & $38.35 \pm 2.45^{\mathrm{a}}$ & $39.32 \pm 1.77^{\mathrm{a} *}$ & $37.00 \pm 2.29^{a}$ \\
\hline MU & $47.86 \pm 2.18^{a}$ & $45.76 \pm 1.98^{\mathrm{a} \star}$ & $47.96 \pm 2.96^{\mathrm{a} \star}$ & $45.82 \pm 2.20^{\mathrm{a} *}$ & $45.55 \pm 2.15^{\mathrm{a} \star}$ & $44.97 \pm 0.34^{a *}$ & $46.95 \pm 2.36^{a}$ \\
\hline PU & $13.73 \pm 1.71^{\mathrm{a}}$ & $15.64 \pm 2.19^{a}$ & $14.53 \pm 2.43^{\mathrm{a}}$ & $15.83 \pm 2.26^{a}$ & $16.1 \pm 1.75^{\mathrm{a}}$ & $15.72 \pm 1.82^{\mathrm{a}}$ & $16.05 \pm 0.58^{a}$ \\
\hline U & $61.59 \pm 0.82^{\mathrm{a}}$ & $60.90 \pm 2.21^{a *}$ & $62.49 \pm 2.06^{\mathrm{a} *}$ & $61.61 \pm 2.44^{\mathrm{a} *}$ & $61.65 \pm 2.45^{\mathrm{a}}$ & $60.68 \pm 1.77^{\mathrm{a} *}$ & $62.99 \pm 2.29^{a}$ \\
\hline $\mathbf{S} / \mathbf{U}$ & $0.62 \pm 0.02^{\mathrm{a}}$ & $0.63 \pm 0.04^{\mathrm{a}}$ & $0.60 \pm 0.05^{\mathrm{a}}$ & $0.63 \pm 0.07^{\mathrm{a}}$ & $0.62 \pm 0.06^{\mathrm{a}}$ & $0.65 \pm 0.05^{\mathrm{a}}$ & $0.59 \pm 0.06^{\mathrm{a}}$ \\
\hline
\end{tabular}

S: sum of saturated fatty acids; $\mathrm{MU}$ : sum of monounsaturated fatty acids; PU: sum of polyunsaturated fatty acids; U: sum of unsaturated fatty acids; S/U: values of ratio saturated/unsaturated fatty acids.

a Means within the same row not followed by the same letter differ significantly $(p<0.05)$.

* Values which resulted significantly different $(p<0.05)$ on comparing the sausages made by artisanal methods with tose made by industrial methods in this sampling point.

\subsection{Analytical methods}

The fat of the samples was extracted following the procedure described by Folch et al. (1957).

In order to determine the total fatty acids, for the formation of methyl esters of the fatty acids which form part of the glycerids and phospholipids the method of transesterification of Shehata et al. (1970) was used.

The free fatty acids were separated from the triglycerides in columns of polypropylene with filling $\mathrm{NH}_{2}$-aminopropyl following the procedure described by Antequera et al. (1994).

For the methylation of the free fatty acids the procedure described by Shehata et al. (1970) with some modifications was used. Immediately after separation from the triglycerides, $2 \mathrm{~mL}$ of a solution $\mathrm{H}_{2} \mathrm{SO}_{4}: \mathrm{CH}_{3} \mathrm{OH}[1: 1,(\mathrm{v} / \mathrm{v})]$ was added to the extract. The mixture was strongly shaken and the free fatty acid methyl esters extraction was carried out using 2 $\mathrm{mL}$ of hexane consecutive twice. The organic phase was separated and dehydrated using $\mathrm{Na}_{2} \mathrm{SO}_{4}$ anhydre. This phase was filtered and evaporated with nitrogen gas. Finally, the dried extract obtained was melted in $2 \mathrm{~mL}$ of hexane.

The identification and quantification of the total and free fatty acids was carried out by gas chromatography techniques, using a chromatograph Hewlett Packard 6890 Series Gc System (Hewlett Packard, Palo Alto, CA, USA) equipped with a Hewlett Packard 7683 Series Injector and a Hewlett Packard 5973 Mass Selective Detector. The chromatographic conditions used are described in Table I.

The separation of the different fatty acids was carried out in a Supelco Omegawax ${ }^{\text {TM }} 250$ Fused Silica Capillary Column; 30 m; 25 mm ID; $0.25 \mu \mathrm{m}$ film thickness (Supelco Park, Bellefonte, PA, USA).

Solutions of a concentration of $500 \mathrm{ppm}$ in hexane of the methyl esters of the following fatty acids: caproic (C6), caprilic (C8), capric (C10), lauric (C12), myristic (C14), palmitic (C16), palmitoleic (C16:1), stearic (C18), oleic (C18:1), linoleic (C18:2), linolenic (C18:3), arachidic (C20) and arachidonic (C20:4) were prepared as patterns. Fatty acids were 
Table III

Evolution of the content of the different fatty acids (expressed as \% of the total fatty acids) during the manufacture of "Chorizo de cebolla" made by industrial methods (means \pm standard deviations of four batches)

\begin{tabular}{|c|c|c|c|c|c|c|c|}
\hline & \multicolumn{7}{|c|}{ Ripening time (days) } \\
\hline & 0 & 2 & 7 & 14 & 21 & 28 & 42 \\
\hline $\mathrm{C}_{10}$ & $0.11 \pm 0.02^{\mathrm{a}}$ & $0.11 \pm 0.02^{\mathrm{a}}$ & $0.11 \pm 0.01^{\mathrm{a}}$ & $0.10 \pm 0.02^{\mathrm{a}}$ & $0.10 \pm 0.01^{\mathrm{a}}$ & $0.10 \pm 0.02^{\mathrm{a}}$ & $0.10 \pm 0.02^{\mathrm{a}}$ \\
\hline $\mathrm{C}_{12}$ & $0.10 \pm 0.02^{\mathrm{a}}$ & $0.10 \pm 0.02^{\mathrm{a}}$ & $0.10 \pm 0.01^{\mathrm{a}}$ & $0.11 \pm 0.01^{\mathrm{a}}$ & $0.10 \pm 0.01^{\mathrm{a}}$ & $0.10 \pm 0.01^{\mathrm{a}}$ & $0.10 \pm 0.01^{\mathrm{a}}$ \\
\hline $\mathrm{C}_{14}$ & $1.20 \pm 0.16^{a}$ & $1.23 \pm 0.14^{\mathrm{a}}$ & $1.23 \pm 0.14^{\mathrm{a}}$ & $1.22 \pm 0.18^{a}$ & $1.25 \pm 0.12^{\mathrm{a}}$ & $1.19 \pm 0.12^{\mathrm{a}}$ & $1.18 \pm 0.14^{\mathrm{a}}$ \\
\hline $\mathrm{C}_{16}$ & $21.88 \pm 1.83^{\mathrm{a}}$ & $21.98 \pm 1.89^{a}$ & $21.90 \pm 1.03^{\mathrm{a}}$ & $21.57 \pm 1.37^{\mathrm{a}}$ & $21.70 \pm 1.06^{\mathrm{a}}$ & $21.82 \pm 1.10^{\mathrm{a}}$ & $21.56 \pm 1.51^{\mathrm{a}}$ \\
\hline $\mathrm{C}_{18}$ & $11.15 \pm 1.45^{\mathrm{a}}$ & $10.97 \pm 1.79^{\mathrm{a}}$ & $11.00 \pm 1.07^{\mathrm{a}}$ & $11.08 \pm 1.43^{\mathrm{a}}$ & $11.34 \pm 1.22^{a}$ & $11.50 \pm 1.36^{a}$ & $11.24 \pm 1.57^{\mathrm{a}}$ \\
\hline $\mathrm{C}_{20}$ & $0.62 \pm 0.28^{\mathrm{a}}$ & $0.55 \pm 0.31^{a}$ & $0.52 \pm 0.26^{a}$ & $0.53 \pm 0.27^{\mathrm{a}}$ & $0.59 \pm 0.29^{a}$ & $0.59 \pm 0.27^{\mathrm{a}}$ & $0.66 \pm 0.21^{\mathrm{a}}$ \\
\hline$C_{16: 1}$ & $2.15 \pm 0.36^{a}$ & $2.16 \pm 0.29^{a}$ & $2.21 \pm 0.13^{\mathrm{a}}$ & $2.14 \pm 0.33^{\mathrm{a}}$ & $2.17 \pm 0.07^{\mathrm{a}}$ & $2.09 \pm 0.13^{a}$ & $2.10 \pm 0.29^{\mathrm{a}}$ \\
\hline $\mathrm{C}_{18: 1}$ & $49.16 \pm 4.47^{\mathrm{a}}$ & $49.08 \pm 3.73^{\mathrm{a}}$ & $48.30 \pm 3.36^{a}$ & $48.80 \pm 5.12^{a}$ & $48.04 \pm 3.35^{\mathrm{a}}$ & $48.17 \pm 3.90^{\mathrm{a}}$ & $48.46 \pm 4.29^{a}$ \\
\hline$C_{18: 2}$ & $13.05 \pm 2.41^{a}$ & $12.84 \pm 1.90^{\mathrm{a}}$ & $13.90 \pm 2.15^{\mathrm{a}}$ & $13.77 \pm 3.06^{a}$ & $14.00 \pm 2.52^{\mathrm{a}}$ & $13.78 \pm 2.74^{a}$ & $13.87 \pm 2.92^{\mathrm{a}}$ \\
\hline $\mathbf{C}_{18.3}$ & $0.58 \pm 0.17^{\mathrm{a}}$ & $0.58 \pm 0.20^{\mathrm{a}}$ & $0.74 \pm 0.08^{\mathrm{a}}$ & $0.64 \pm 0.09^{a}$ & $0.71 \pm 0.06^{\mathrm{a}}$ & $0.66 \pm 0.09^{a}$ & $0.70 \pm 0.10^{\mathrm{a}}$ \\
\hline $\mathrm{C}_{20: 4}$ & - & - & - & $0.03 \pm 0.06^{\mathrm{a}}$ & - & - & $0.04 \pm 0.04^{\mathrm{a}}$ \\
\hline S & $35.11 \pm 3.13^{\mathrm{a}}$ & $34.99 \pm 3.40^{\mathrm{a}}$ & $34.85 \pm 2.02^{\mathrm{a}}$ & $34.65 \pm 2.73^{\mathrm{a}}$ & $35.07 \pm 2.27^{\mathrm{a}}$ & $35.31 \pm 2.38^{\mathrm{a}}$ & $34.86 \pm 3.03^{\mathrm{a}}$ \\
\hline MU & $51.31 \pm 4.16^{a}$ & $51.24 \pm 3.56^{a}$ & $50.52 \pm 3.27^{\mathrm{a}}$ & $50.95 \pm 4.81^{\mathrm{a}}$ & $50.21 \pm 3.37^{\mathrm{a}}$ & $50.26 \pm 3.80^{a}$ & $50.56 \pm 4.04^{\mathrm{a}}$ \\
\hline PU & $13.64 \pm 2.39^{\mathrm{a}}$ & $13.42 \pm 1.95^{\mathrm{a}}$ & $14.64 \pm 2.17^{\mathrm{a}}$ & $14.45 \pm 3.10^{\mathrm{a}}$ & $14.46 \pm 2.2^{\mathrm{a}}$ & $14.45 \pm 2.82^{\mathrm{a}}$ & $14.61 \pm 3.01^{\mathrm{a}}$ \\
\hline U & $64.95 \pm 3.20^{\mathrm{a}}$ & $64.66 \pm 2.84^{a}$ & $65.16 \pm 2.02^{a}$ & $65.39 \pm 2.78^{a}$ & $64.68 \pm 2.16^{\mathrm{a}}$ & $64.71 \pm 2.38^{\mathrm{a}}$ & $65.17 \pm 3.03^{\mathrm{a}}$ \\
\hline $\mathbf{S} / \mathbf{U}$ & $0.62 \pm 0.07^{\mathrm{a}}$ & $0.63 \pm 0.08^{\mathrm{a}}$ & $0.65 \pm 0.05^{\mathrm{a}}$ & $0.63 \pm 0.06^{\mathrm{a}}$ & $0.62 \pm 0.05^{\mathrm{a}}$ & $0.65 \pm 0.06^{\mathrm{a}}$ & $0.59 \pm 0.08^{\mathrm{a}}$ \\
\hline
\end{tabular}

S: sum of saturated fatty acids; $\mathrm{MU}$ : sum of monounsaturated fatty acids; PU: sum of polyunsaturated fatty acids; $\mathrm{U}$ : sum of unsaturated fatty acids; $\mathrm{S} / \mathrm{U}$ : values of ratio saturated/unsaturated fatty acids.

a Means within the same row not followed by the same letter differ significantly $(p<0.05)$.

purchased from Sigma Chemical Co. (Saint Louis, MO, USA).

For the quantification of the different fatty acids, using external standards, calibration graphs were prepared using different concentrations of each methyl ester.

All the samples and patterns were injected at least in duplicate. Repeatability tests were done, injecting a pattern and a sample consecutively six times a day, and a reproductibility test, injecting the patter and the sample twice a day for three days, in the same experimental conditions. Significant differences $(p<0.05)$ between the results in both tests were not found.

\subsection{Statistic analysis}

For the comparison of the different parameters studied throughout the ripening period and between the artisanal and industrial procedures, a variance analysis (ANOVA) was carried out with an interval of reliability of $95 \% \quad(p<0.05)$ using the computer program Statistica ${ }^{\odot} 5.1$ for Windows (Statsoft Inc., Tulsa, OK, USA).

\section{RESULTS AND DISCUSSION}

\subsection{Total fatty acids}

Tables II and III show the evolution of the total fatty acids (expressed as \%) during the ripening of "Chorizo de cebolla" batches made by artisanal and industrial methods, respectively. High standard deviation values were observed in each sampling point both in artisanal and industrial sausages. This great variability between the batches of a same manufacture type may be due to the different geographical origin and to the different composition of the fat used in the manufacture of the different batches.

The values, expressed as percentage, of the different fatty acids constitutive of glycerides and phospholypids of the fat in the 'chorizo de cebolla' stayed practically constant throughout the whole ripening process. The main identified fatty acids in both types of sausages were in decreasing order of concentration: oleic acid, palmitic acid, linoleic acid, stearic acid and palmitoleic acid. This profile coincides basically with that found by other authors 
Table IV

Evolution of the content of the different free fatty acids (expressed as $\mathbf{m g} / \mathbf{1 0 0} \mathrm{g}$ of fat) during the manufacture of "Chorizo de cebolla" made by artisanal methods (means \pm standard deviations of four batches).

\begin{tabular}{|c|c|c|c|c|c|c|c|}
\hline \multicolumn{8}{|c|}{ Ripening time (Days) } \\
\hline & 0 & 2 & 7 & 14 & 21 & 28 & 42 \\
\hline $\mathrm{C}_{10}$ & $1.09 \pm 0.31^{a}$ & $1.91 \pm 0.80^{\mathrm{a}}$ & $2.05 \pm 0.54^{\mathrm{a}}$ & $2.37 \pm 0.87^{\mathrm{ab}}$ & $2.96 \pm 1.55^{\mathrm{ab}}$ & $4.31 \pm 1.50^{\mathrm{b}}$ & $4.49 \pm 2.11^{\mathrm{b}}$ \\
\hline $\mathrm{C}_{12}$ & $1.66 \pm 0.54^{\mathrm{a}}$ & $2.32 \pm 0.89^{\mathrm{a}}$ & $2.44 \pm 0.72^{\mathrm{a}}$ & $2.63 \pm 0.60^{\mathrm{ab}}$ & $2.96 \pm 1.76^{\mathrm{ab}}$ & $6.09 \pm 3.55^{\mathrm{bc}}$ & $7.04 \pm 5.06^{c}$ \\
\hline $\mathrm{C}_{14}$ & $5.86 \pm 1.92^{\mathrm{a}}$ & $10.18 \pm 4.05^{\mathrm{ab}}$ & $13.20 \pm 4.57^{\mathrm{ab}}$ & $18.01 \pm 8.02^{\mathrm{ab}}$ & $21.35 \pm 12.38^{\mathrm{ab}}$ & $34.01 \pm 12.34^{\mathrm{ab}}$ & $48.42 \pm 24.39^{b}$ \\
\hline $\mathrm{C}_{16}$ & $81.37 \pm 58.97^{\mathrm{a}}$ & $140.66 \pm 78.21^{a}$ & $231.17 \pm 145.08^{\mathrm{a}}$ & $328.43 \pm 221.54^{\mathrm{ab}}$ & $354.33 \pm 281.84^{\mathrm{ab}}$ & $496.48 \pm 291.68^{\mathrm{ab}}$ & $806.89 \pm 542.55^{\mathrm{a}}$ \\
\hline $\mathrm{C}_{18}$ & $42.96 \pm 29.53^{\mathrm{a}}$ & $78.44 \pm 45.32^{\mathrm{ab}}$ & $142.18 \pm 83.77^{\mathrm{abc}}$ & $223.79 \pm 153.59^{\mathrm{abc}}$ & $228.20 \pm 184.42^{\mathrm{abc}}$ & $330.99 \pm 262.99^{b c}$ & $388.45 \pm 285.77^{\circ}$ \\
\hline $\mathrm{C}_{20}$ & $1.75 \pm 2.02^{\mathrm{a}}$ & $4.58 \pm 3.10^{\mathrm{a}}$ & $9.89 \pm 4.16^{\mathrm{ab}}$ & $14.12 \pm 5.6^{\mathrm{abc}} 0$ & $19.84 \pm 12.73^{\text {abc }}$ & $27.14 \pm 10.63^{\text {bc }}$ & $32.38 \pm 17.47^{\circ}$ \\
\hline$C_{16: 1}$ & $9.56 \pm 4.33^{\mathrm{a}}$ & $15.50 \pm 7.00^{\mathrm{a}}$ & $20.91 \pm 9.38^{\mathrm{a}}$ & $30.64 \pm 19.26^{\mathrm{a}}$ & $40.21 \pm 28.65^{\mathrm{a}}$ & $50.37 \pm 23.34^{\mathrm{a}}$ & $59.35 \pm 29.01^{\mathrm{at}}$ \\
\hline$C_{18: 1}$ & $169.28 \pm 104.56^{\mathrm{a}}$ & $294.29 \pm 120.81^{a}$ & $490.83 \pm 194.77^{\mathrm{ab}}$ & $637.22 \pm 165.26^{\text {ab }}$ & $824.90 \pm 500.50^{a b}$ & $1028.03 \pm 347.68^{\mathrm{ab}}$ & $1285.24 \pm 449.42^{\circ}$ \\
\hline$C_{18: 2}$ & $140.24 \pm 60.57^{\mathrm{a}}$ & $273.13 \pm 154.52^{\mathrm{ab}}$ & $394.26 \pm 271.00^{\mathrm{ab}}$ & $385.81 \pm 236.98^{\mathrm{abc}}$ & $722.35 \pm 441.59^{\text {bod }}$ & $861.43 \pm 344.52^{\mathrm{cd}}$ & $983.12 \pm 349.56^{d}$ \\
\hline$C_{18.3}$ & $5.85 \pm 1.71^{\mathrm{a}}$ & $13.44 \pm 8.12^{\mathrm{a}}$ & $26.52 \pm 24.27^{\mathrm{ab}}$ & $32.67 \pm 21.89^{\mathrm{ab}}$ & $44.86 \pm 26.02^{\mathrm{bc}}$ & $56.21 \pm 22.30^{\text {bc }}$ & $65.30 \pm 28.07^{\circ}$ \\
\hline$C_{20: 4}$ & $0.21 \pm 0.31^{\mathrm{a}}$ & $1.31 \pm 0.86^{\mathrm{a}}$ & $3.62 \pm 3.02^{\mathrm{ab}}$ & $3.68 \pm 2.74^{\mathrm{ab}}$ & $5.11 \pm 3.50^{\mathrm{ab}}$ & $6.69 \pm 2.08^{\mathrm{b}}$ & $7.22 \pm 2.46^{b *}$ \\
\hline$\Sigma$ FFA & $459.83 \pm 243.06^{\mathrm{a}}$ & $835.76 \pm 384.13^{\mathrm{ab}}$ & $1337.07 \pm 618.43^{\mathrm{ab}}$ & $1679.37 \pm 718.47^{\mathrm{abc}}$ & $2266.17 \pm 1466.17^{\text {abc }}$ & $2901.75 \pm 1262.62^{\mathrm{bc}}$ & $3687.9 \pm 1670.01^{\circ}$ \\
\hline s & $134.69 \pm 90.14^{\mathrm{a}}$ & $238.09 \pm 128.85^{\mathrm{a}}$ & $400.93 \pm 237.30^{\mathrm{a}}$ & $589.35 \pm 388.63^{\mathrm{ab}}$ & $629.64 \pm 494.22^{\mathrm{ab}}$ & $899.02 \pm 612.37^{\mathrm{ab}}$ & $1287.67 \pm 821.64$ \\
\hline MU & $178.84 \pm 108.16^{\mathrm{a}}$ & $309.79 \pm 127.72^{\mathrm{ab}}$ & $511.74 \pm 203.24^{\mathrm{ab}}$ & $667.86 \pm 180.97^{\mathrm{ab}}$ & $864.21 \pm 529.04^{\mathrm{ab}}$ & $1078.4 \pm 370.57^{\mathrm{ab}}$ & $1344.59 \pm 495.85$ \\
\hline PU & $146.3 \pm 62.02^{\mathrm{a}}$ & $287.88 \pm 162.51^{\mathrm{ab}}$ & $424.4 \pm 298.10^{\mathrm{ab}}$ & $422.16 \pm 260.95^{\mathrm{abc}}$ & $772.32 \pm 467.47^{\text {bod }}$ & $924.33 \pm 365.39^{\text {cd }}$ & $1055.64 \pm 379.27$ \\
\hline U & $325.14 \pm 156.00^{\mathrm{a}}$ & $597.67 \pm 283.35^{\mathrm{a}}$ & $936.14 \pm 454.30^{\mathrm{ab}}$ & $1090.02 \pm 393.47^{\mathrm{ab}}$ & $1636.53 \pm 984.21^{\mathrm{ab}}$ & $2002.73 \pm 706.48^{b}$ & $2400.23 \pm 873.13^{\circ}$ \\
\hline$s / U$ & $0.41 \pm 0.11^{\mathrm{a}}$ & $0.40 \pm 0.14^{\mathrm{a}}$ & $0.43 \pm 0.19^{\mathrm{a}}$ & $0.54 \pm 0.20^{\mathrm{a}}$ & $0.38 \pm 0.09^{\mathrm{a}}$ & $0.45 \pm 0.15^{a}$ & $0.54 \pm 0.16^{\mathrm{a}}$ \\
\hline
\end{tabular}

S: sum of saturated fatty acids; $\mathrm{MU}$ : sum of monounsaturated fatty acids; $\mathrm{PU}$ : sum of polyunsaturated fatty acids; $\mathrm{U}$ : sum of unsaturated fatty acids; S/U: values of ratio saturated/unsaturated fatty acids.

${ }^{a d d}$ Means within the same row not followed by the same letter differ significantly $(p<0.05)$.

* Values which resulted significantly different $(p<0.05)$ on comparing the sausages made by artisanal methods with tose made by industrial methods in this sampling point. 
Table $\mathrm{V}$

Evolution of the content of the different free fatty acids (expressed as $\mathrm{mg} / 100 \mathrm{~g}$ of fat) during the manufacture of "Chorizo de cebolla" made by industrial (means \pm standard deviations of four batches).

\begin{tabular}{|c|c|c|c|c|c|c|c|}
\hline & \multicolumn{7}{|c|}{ Ripening time (days) } \\
\hline & 0 & 2 & 7 & 14 & 21 & 28 & 42 \\
\hline $\mathrm{C}_{10}$ & $1.28 \pm 0.76^{a}$ & $1.59 \pm 0.97^{\mathrm{a}}$ & $2.02 \pm 1.1^{\mathrm{ab}}$ & $1.70 \pm 1.07^{\mathrm{ab}}$ & $1.93 \pm 0.86^{\mathrm{ab}}$ & $3.80 \pm 2.14^{\mathrm{bc}}$ & $5.91 \pm 3.50^{\circ}$ \\
\hline $\mathrm{C}_{12}$ & $3.04 \pm 1.96^{\mathrm{a}}$ & $2.03 \pm 0.83^{\mathrm{a}}$ & $2.61 \pm 1.09^{\mathrm{ab}}$ & $2.56 \pm 1.48^{\mathrm{ab}}$ & $3.12 \pm 1.47^{\mathrm{ab}}$ & $5.96 \pm 3.11^{\mathrm{bc}}$ & $7.31 \pm 4.96^{c}$ \\
\hline $\mathrm{C}_{14}$ & $8.20 \pm 5.31^{a}$ & $8.97 \pm 5.76^{\mathrm{ab}}$ & $13.25 \pm 11.03^{\text {ab }}$ & $16.31 \pm 10.08^{\mathrm{ab}}$ & $20.42 \pm 13.69^{\mathrm{ab}}$ & $48.38 \pm 35.08^{\mathrm{bc}}$ & $81.08 \pm 89.76^{\circ}$ \\
\hline $\mathrm{C}_{16}$ & $97.31 \pm 55.19^{\mathrm{a}}$ & $113.34 \pm 65.06^{a}$ & $146.34 \pm 99.5^{\mathrm{a}}$ & $181.53 \pm 93.85^{\mathrm{a}}$ & $243.82 \pm 149.53^{\mathrm{a}}$ & $584.94 \pm 432.66^{\mathrm{a}}$ & $743.71 \pm 605.03^{\mathrm{a}}$ \\
\hline $\mathrm{C}_{18}$ & $49.71 \pm 30.91^{a}$ & $66.18 \pm 46.73^{\mathrm{a}}$ & $106.18 \pm 77.13^{\text {ab }}$ & $115.80 \pm 55.32^{\mathrm{ab}}$ & $138.29 \pm 70.92^{\mathrm{ab}}$ & $383.42 \pm 213.57^{\circ c}$ & $501.17 \pm 304.75^{\circ}$ \\
\hline $\mathrm{C}_{20}$ & $0.44 \pm 0.19^{\mathrm{a}}$ & $0.49 \pm 0.18^{a}$ & $1.80 \pm 2.39^{\mathrm{a}}$ & $10.84 \pm 6.03^{\mathrm{a}}$ & $11.91 \pm 6.60^{\mathrm{a}}$ & $37.63 \pm 29.84^{\mathrm{b}}$ & $42.67 \pm 26.41^{\mathrm{b}}$ \\
\hline$C_{16: 1}$ & $12.94 \pm 9.74^{\mathrm{a}}$ & $16.77 \pm 12.88^{\mathrm{a}}$ & $40.43 \pm 55.92^{\mathrm{ab}}$ & $27.04 \pm 20.94^{\mathrm{ab}}$ & $35.43 \pm 25.94^{\mathrm{ab}}$ & $80.94 \pm 67.04^{\text {bc }}$ & $128.77 \pm 120.15^{\circ}$ \\
\hline$C_{18: 1}$ & $221.04 \pm 146.61^{a}$ & $275.43 \pm 151.92^{\mathrm{a}}$ & $396.61 \pm 253.54^{\mathrm{a}}$ & $538.99 \pm 333.87^{\mathrm{a}}$ & $687.66 \pm 451.94^{\mathrm{a}}$ & $1600.68 \pm 1164.61^{b}$ & $2127.10 \pm 1475.69^{\circ}$ \\
\hline $\mathbf{C}_{18: 2}$ & $160.23 \pm 68.16^{a}$ & $196.15 \pm 85.45^{\mathrm{a}}$ & $303.68 \pm 166.66^{\mathrm{a}}$ & $354.49 \pm 133.42^{\mathrm{a}}$ & $471.13 \pm 222.86^{\mathrm{a}}$ & $1065.54 \pm 612.26^{b}$ & $1414.55 \pm 508.01^{b}$ \\
\hline $\mathrm{C}_{18.3}$ & $6.10 \pm 1.87^{\mathrm{a}}$ & $9.85 \pm 2.14^{\mathrm{a}}$ & $12.19 \pm 7.71^{\mathrm{a}}$ & $19.98 \pm 10.30^{\mathrm{a}}$ & $33.74 \pm 16.31^{\mathrm{a}}$ & $85.85 \pm 42.89^{b}$ & $89.94 \pm 34.46^{\mathrm{b}}$ \\
\hline $\mathbf{C}_{20: 4}$ & $0.05 \pm 0.03^{\mathrm{a}}$ & $0.06 \pm 0.02^{\mathrm{a}}$ & $1.85 \pm 2.22^{\mathrm{ab}}$ & $2.74 \pm 2.33^{\mathrm{ab}}$ & $6.27 \pm 4.12^{\mathrm{bc}}$ & $10.16 \pm 6.55^{\mathrm{cd}}$ & $14.64 \pm 8.37^{d}$ \\
\hline$\Sigma$ FFA & $560.34 \pm 317.28^{\mathrm{a}}$ & $690.86 \pm 362.64^{a}$ & $1026.96 \pm 657.16^{\mathrm{a}}$ & $1270.98 \pm 640.54^{a}$ & $1653.72 \pm 957.58^{\mathrm{a}}$ & $3917.3 \pm 2588.97^{b}$ & $5157.09 \pm 3673.69^{\circ}$ \\
\hline s & $159.98 \pm 92.63^{\mathrm{a}}$ & $192.6 \pm 118.12^{\mathrm{a}}$ & $270.42 \pm 224.12^{\mathrm{ab}}$ & $328.74 \pm 166.67^{\mathrm{ab}}$ & $419.49 \pm 239.67^{\mathrm{ab}}$ & $1064.13 \pm 679.66^{b}$ & $1381.85 \pm 1279.14^{\circ}$ \\
\hline MU & $233.98 \pm 156.33^{\mathrm{a}}$ & $292.2 \pm 164.61^{\mathrm{a}}$ & $437.04 \pm 297.91^{\mathrm{a}}$ & $566.03 \pm 354.62^{\mathrm{a}}$ & $723.09 \pm 479.50^{\mathrm{a}}$ & $1681.62 \pm 1231.58^{\mathrm{b}}$ & $2255.87 \pm 1505.79^{b}$ \\
\hline PU & $166.38 \pm 69.78^{\mathrm{a}}$ & $206.06 \pm 86.60^{\mathrm{a}}$ & $317.72 \pm 165.45^{\mathrm{a}}$ & $376.21 \pm 144.08^{\mathrm{a}}$ & $511.14 \pm 243.23^{\mathrm{a}}$ & $1161.55 \pm 658.22^{b}$ & $1519.13 \pm 609.13^{b}$ \\
\hline U & $400.36 \pm 224.72^{\mathrm{a}}$ & $498.26 \pm 245.36^{a}$ & $754.76 \pm 421.22^{\mathrm{a}}$ & $942.24 \pm 473.73^{\mathrm{a}}$ & $1234.23 \pm 719.39^{a}$ & $2843.17 \pm 1869.88^{b}$ & $3775.14 \pm 1921.04^{\circ}$ \\
\hline$S / U$ & $0.40 \pm 0.03^{\mathrm{a}}$ & $0.39 \pm 0.05^{\mathrm{a}}$ & $0.36 \pm 0.09^{\mathrm{a}}$ & $0.35 \pm 0.01^{\mathrm{a}}$ & $0.34 \pm 0.00^{\mathrm{a}}$ & $0.38 \pm 0.03^{\mathrm{a}}$ & $0.36 \pm 0.06^{a}$ \\
\hline
\end{tabular}

$\mathrm{S}$ : sum of saturated fatty acids; $\mathrm{MU}$ : sum of monounsaturated fatty acids; PU: sum of polyunsaturated fatty acids; U: sum of unsaturated fatty acids; S/U: ratio values saturated/unsaturated fatty acids.

a:d Means within the same row not followed by the same letter differ significantly $(p<0.05)$. 
Table VI

Evolution of the content of the different free fatty acids (expressed as $\%$ of the total free fatty acids) during the manufacture of "Chorizo de cebolla" made by artisanal methods (means \pm standard deviations of four batches).

\begin{tabular}{|c|c|c|c|c|c|c|c|}
\hline & \multicolumn{7}{|c|}{ Ripening time (days) } \\
\hline & 0 & 2 & 7 & 14 & 21 & 28 & 42 \\
\hline $\mathrm{C}_{10}$ & $0.24 \pm 0.11^{\mathrm{a}}$ & $0.23 \pm 0.17^{a}$ & $0.15 \pm 0.08^{a}$ & $0.14 \pm 0.04^{\mathrm{a}}$ & $0.13 \pm 0.06^{\mathrm{a}}$ & $0.15 \pm 0.03^{\mathrm{a}}$ & $0.12 \pm 0.03^{\mathrm{a}}$ \\
\hline$C_{12}$ & $0.37 \pm 0.23^{\mathrm{a}}$ & $0.28 \pm 0.22^{a}$ & $0.18 \pm 0.10^{\mathrm{a}}$ & $0.16 \pm 0.03^{\mathrm{a}}$ & $0.14 \pm 0.02^{a}$ & $0.21 \pm 0.12^{\mathrm{a}}$ & $0.19 \pm 0.09^{a}$ \\
\hline $\mathrm{C}_{14}$ & $1.27 \pm 0.39^{\mathrm{a}}$ & $1.21 \pm 0.34^{\mathrm{a}}$ & $0.99 \pm 0.31^{a}$ & $1.07 \pm 0.25^{\mathrm{a}}$ & $0.94 \pm 0.27^{\mathrm{a}}$ & $1.17 \pm 0.15^{\mathrm{a}}$ & $1.31 \pm 0.64^{\mathrm{a}}$ \\
\hline$C_{16}$ & $17.65 \pm 3.99^{\mathrm{a}}$ & $16.83 \pm 4.41^{\mathrm{a}}$ & $17.29 \pm 4.73^{\mathrm{a}}$ & $19.56 \pm 4.59^{\mathrm{a}}$ & $15.64 \pm 2.59^{a}$ & $17.10 \pm 2.76^{a}$ & $21.88 \pm 6.22^{\circ}$ \\
\hline$C_{18}$ & $9.34 \pm 2.17^{\mathrm{a}}$ & $9.39 \pm 2.54^{a}$ & $10.63 \pm 2.94^{\mathrm{a}}$ & $13.33 \pm 2.80^{\mathrm{a}}$ & $10.07 \pm 1.75^{\mathrm{a}}$ & $11.41 \pm 3.46^{a}$ & $10.54 \pm 3.36^{\circ}$ \\
\hline $\mathrm{C}_{20}$ & $0.38 \pm 0.34^{\mathrm{a}}$ & $0.55 \pm 0.28^{\mathrm{a}}$ & $0.74 \pm 0.36^{\mathrm{a}}$ & $0.85 \pm 0.34^{\mathrm{a}}$ & $0.88 \pm 0.25^{\mathrm{a}}$ & $0.94 \pm 0.22^{\mathrm{a}}$ & $0.88 \pm 0.26^{a}$ \\
\hline$C_{16: 1}$ & $2.08 \pm 0.48^{a}$ & $1.87 \pm 0.15^{\mathrm{ab} *}$ & $1.56 \pm 0.28^{\mathrm{bc*}}$ & $1.82 \pm 0.37^{\mathrm{bc}}$ & $1.77 \pm 0.24^{\circ}$ & $1.75 \pm 0.26^{c}$ & $1.61 \pm 0.21^{\circ}$ \\
\hline$C_{18: 1}$ & $36.81 \pm 6.14^{\mathrm{a}}$ & $35.21 \pm 3.73^{\mathrm{a}}$ & $36.71 \pm 5.01^{\mathrm{a}}$ & $37.98 \pm 8.37^{\mathrm{a}}$ & $36.40 \pm 3.73^{\mathrm{a}}$ & $35.43 \pm 3.88^{a}$ & $34.85 \pm 4.17^{a}$ \\
\hline $\mathrm{C}_{18: 2}$ & $30.50 \pm 10.68^{a}$ & $32.68 \pm 9.71^{a}$ & $29.49 \pm 9.24^{\mathrm{a}}$ & $26.97 \pm 9.64^{\mathrm{a}}$ & $31.87 \pm 6.14^{\mathrm{a}}$ & $29.69 \pm 5.47^{a}$ & $26.66 \pm 2.95^{\mathrm{a}}$ \\
\hline $\mathrm{C}_{18.3}$ & $1.26 \pm 0.54^{\mathrm{a}}$ & $1.62 \pm 0.54^{\mathrm{a}}$ & $1.98 \pm 0.99^{\mathrm{a}}$ & $1.95 \pm 1.18^{\mathrm{a}}$ & $1.98 \pm 0.91^{\mathrm{a}}$ & $1.95 \pm 0.61^{\mathrm{a}}$ & $1.77 \pm 0.19^{\mathrm{a}}$ \\
\hline$C_{20: 4}$ & $0.05 \pm 0.04^{\mathrm{a}}$ & $0.16 \pm 0.08^{\mathrm{ab}}$ & $0.27 \pm 0.14^{b}$ & $0.22 \pm 0.16^{\mathrm{ab}}$ & $0.23 \pm 0.18^{b}$ & $0.23 \pm 0.09^{b}$ & $0.20 \pm 0.05^{\mathrm{a}}$ \\
\hline S & $29.33 \pm 7.26^{a}$ & $28.05 \pm 7.91^{\mathrm{a}}$ & $29.98 \pm 9.15^{\mathrm{a}}$ & $35.10 \pm 4.93^{\mathrm{a}}$ & $27.78 \pm 4.93^{\mathrm{a}}$ & $30.99 \pm 6.73^{a}$ & $34.91 \pm 9.54^{\mathrm{a}}$ \\
\hline MU & $38.89 \pm 6.18^{a}$ & $37.06 \pm 3.80^{\mathrm{a}}$ & $38.27 \pm 5.07^{\mathrm{a}}$ & $39.73 \pm 8.12^{\mathrm{a}}$ & $38.17 \pm 3.48^{\mathrm{a}}$ & $37.16 \pm 4.07^{a}$ & $36.46 \pm 4.20^{\circ}$ \\
\hline PU & $31.82 \pm 11.76^{a}$ & $34.45 \pm 10.05^{\mathrm{a}}$ & $31.74 \pm 10.09^{\mathrm{a}}$ & $28.63 \pm 10.66^{a}$ & $34.08 \pm 7.20^{\mathrm{a}}$ & $31.87 \pm 5.71^{\mathrm{a}}$ & $28.63 \pm 3.42^{2}$ \\
\hline U & $70.71 \pm 5.71^{\mathrm{a}}$ & $71.51 \pm 7.48^{\mathrm{a}}$ & $70.01 \pm 8.16^{a}$ & $68.36 \pm 8.71^{a}$ & $72.25 \pm 10.35^{a}$ & $69.04 \pm 6.07^{\mathrm{a}}$ & $65.09 \pm 7.12^{2}$ \\
\hline
\end{tabular}

S: sum of saturated fatty acids; $\mathrm{MU}$ : sum of monounsaturated fatty acids; PU: sum of polyunsaturated fatty acids; $\mathrm{U}$ : sum of unsaturated fatty acids.

a-c Means within the same row not followed by the same letter differ significantly $(p<0.05)$.

* Values which resulted significantly different $(p<0.05)$ on comparing the sausages made by artisanal methods with tose made by industrial methods in this sampling point.

in pork fat (Cava et al., 1997; García Regueiro and Díaz, 1997; Davenel et al., 1999).

When compared both types of sausages, significant difference $(p<0.05)$ in the content of monounsaturated and saturated acids were found.

The artisanal made sausages showed percentages of saturated fatty acids of about $38 \%$, palmitic acid and stearic acid were in the majoritary being a percentage of the total fatty acids about 23 and $13 \%$, respectively. In the unsaturated fatty acids, which represent about $61 \%$, the monounsaturated were in the majoritary with a value around $45 \%$, especially the oleic acid which represented about $44 \%$.

The industrially manufactured sausages showed percentages significantly lower of saturated fatty acids, values around $35 \%$, the palmitic $(21 \%)$ and the stearic $(11 \%)$ acids being also in the majoritary saturated fatty acids. Consequently, the content in unsaturated fatty acids was significantly higher, values around $65 \%$, owing to the higher content of oleic acid (48\%) and palmitoleic acid (2\%).

The contents in polyunsaturated fatty acids (around $15 \%$ of the total) were similar in both types of sausages, the linoleic being in the majoritary with average values around $14 \%$. The arachidonic acid was the less abundant fatty acid, not being detected in some sampling points both in the artisanal and industrial batches.

Fat in pork is the most variable component in the composition and depends on sex, dietary fat composition, slaughtered weight, anatomical location, backfat thickness, etc. (Wood et al., 1989; Leszczynski et al., 1992; Lluch et al., 1993; Palanska et al., 1993; Pfalzgraf et al., 1995; Scheeder et al., 2000). The quantitative ratio between saturated and unsaturated fatty acids determines the consistence of the fats; the least consistent and softer fats presented a higher content of oleic acid than the harder ones which usually have a higher content of stearic acid. This ratio between saturated and unsaturated fatty acids also determine the sensibility of the fat to the selfoxidative rancidity, the greater it is the higher is the degree of unsaturation.

The difference we have observed in the content of saturated and unsaturated fatty acids between both types of sausages, could be explained by the 
Table VII

Evolution of the content of the different free fatty acids (expressed as $\%$ of the total free fatty acids) during the manufacture of "Chorizo de cebolla" made by industrial methods (means \pm standard deviations of four batches).

\begin{tabular}{|c|c|c|c|c|c|c|c|}
\hline & \multicolumn{7}{|c|}{ Ripening time (days) } \\
\hline & 0 & 2 & 7 & 14 & 21 & 28 & 42 \\
\hline $\mathrm{C}_{10}$ & $0.22 \pm 0.13^{\mathrm{a}}$ & $0.23 \pm 0.13^{\mathrm{a}}$ & $0.20 \pm 0.15^{\mathrm{a}}$ & $0.14 \pm 0.06^{\mathrm{ab}}$ & $0.11 \pm 0.05^{\mathrm{ab}}$ & $0.11 \pm 0.03^{b}$ & $0.12 \pm 0.05^{b}$ \\
\hline $\mathrm{C}_{12}$ & $0.55 \pm 0.29^{\mathrm{a}}$ & $0.29 \pm 0.09^{b}$ & $0.25 \pm 0.10^{\mathrm{b}}$ & $.04^{b}$ & $\pm 0.03^{\mathrm{b}}$ & $.04^{b}$ & $0.15 \pm 0.02^{b}$ \\
\hline $\mathrm{C}_{14}$ & $1.46 \pm 0.14^{\mathrm{a}}$ & $1.30 \pm 0.22^{\mathrm{a}}$ & $1.29 \pm 0.13^{\mathrm{a}}$ & $1.28 \pm 0.21^{\mathrm{a}}$ & $1.23 \pm 0.19^{\mathrm{a}}$ & $1.24 \pm 0.16^{\mathrm{a}}$ & $1.57 \pm 0.51^{\mathrm{a}}$ \\
\hline $\mathrm{C}_{16}$ & $17.37 \pm 0.20^{\mathrm{a}}$ & $16.41 \pm 0.87^{\mathrm{a}}$ & $14.25 \pm 0.68^{\mathrm{a}}$ & $14.27 \pm 0.51^{\mathrm{a}}$ & $14.74 \pm 0.72^{\mathrm{a}}$ & $14.97 \pm 1.66^{\mathrm{a}}$ & $14.42 \pm 0.72^{\mathrm{a}}$ \\
\hline $\mathrm{C}_{18}$ & $8.87 \pm 1.24^{\mathrm{a}}$ & $9.58 \pm 2.02^{\mathrm{a}}$ & $10.35 \pm 3.33^{\mathrm{a}}$ & $9.10 \pm 0.38^{\mathrm{a}}$ & $8.36 \pm 0.88^{\mathrm{a}}$ & $9.81 \pm 1.37^{\mathrm{a}}$ & $9.72 \pm 1.15^{\mathrm{a}}$ \\
\hline $\mathrm{C}_{20}$ & $0.08 \pm 0.02^{\mathrm{a}}$ & $0.07 \pm 0.01^{\mathrm{ab}}$ & $17 \pm 0.57^{\mathrm{ab}}$ & $0.85 \pm 0.14^{a}$ & $0.72 \pm 0.07^{\mathrm{ab}}$ & $0.96 \pm 0.39^{b}$ & $0.82 \pm 0.10^{\mathrm{a}}$ \\
\hline$C_{16: 1}$ & $2.31 \pm 0.86^{\mathrm{a}}$ & $2.43 \pm 0.98^{\mathrm{a}}$ & $3.94 \pm 2.03^{\mathrm{a}}$ & $2.12 \pm 1.01^{b}$ & $2.14 \pm 0.47^{\circ}$ & $2.07 \pm 0.67^{\circ}$ & $2.49 \pm 0.60^{\mathrm{b}}$ \\
\hline $\mathrm{C}_{18: 1}$ & $39.44 \pm 7.08^{\mathrm{a}}$ & $39.89 \pm 6.44^{\mathrm{a}}$ & $38.62 \pm 1.67^{\mathrm{a}}$ & $42.37 \pm 6.52^{\mathrm{a}}$ & $41.58 \pm 4.94^{\mathrm{a}}$ & $40.97 \pm 2.99^{\mathrm{a}}$ & $41.25 \pm 2.21^{\mathrm{a}}$ \\
\hline$C_{18: 2}$ & $28.60 \pm 8.76^{\mathrm{a}}$ & $28.41 \pm 8.06^{\mathrm{a}}$ & $29.57 \pm 5.19^{\mathrm{a}}$ & $27.86 \pm 7.08^{\mathrm{a}}$ & $28.49 \pm 5.03^{\mathrm{a}}$ & $27.27 \pm 3.72^{\mathrm{a}}$ & $27.43 \pm 4.09^{\mathrm{a}}$ \\
\hline $\mathrm{C}_{18.3}$ & $1.09 \pm 0.47^{\mathrm{a}}$ & $1.37 \pm 0.94^{\mathrm{a}}$ & $1.19 \pm 0.94^{\mathrm{a}}$ & $1.57 \pm 0.59^{\mathrm{a}}$ & $2.04 \pm 0.32^{\mathrm{a}}$ & $2.20 \pm 0.81^{\mathrm{a}}$ & $1.74 \pm 0.78^{\mathrm{a}}$ \\
\hline $\mathrm{C}_{20: 4}$ & $0.01 \pm 0.00^{\mathrm{a}}$ & $0.01 \pm 0.00^{\mathrm{a}}$ & $0.18 \pm 0.26^{b}$ & $0.21 \pm 0.17^{b}$ & $0.38 \pm 0.05^{b}$ & $0.26 \pm 0.02^{\mathrm{b}}$ & $0.28 \pm 0.10^{b}$ \\
\hline s & $28.55 \pm 1.32^{\mathrm{a}}$ & $27.89 \pm 2.72^{\mathrm{a}}$ & $26.51 \pm 3.76^{\mathrm{a}}$ & $25.84 \pm 0.75^{\mathrm{a}}$ & $25.37 \pm 0.23^{\mathrm{a}}$ & $27.23 \pm 1.61^{\mathrm{a}}$ & $26.80 \pm 2.57^{\mathrm{a}}$ \\
\hline MU & $41.77 \pm 7.94^{\mathrm{a}}$ & $42.32 \pm 7.29^{\mathrm{a}}$ & $42.56 \pm 3.26^{\mathrm{a}}$ & $44.49 \pm 7.26^{a}$ & $43.72 \pm 5.50^{\mathrm{a}}$ & $43.04 \pm 3.47^{\mathrm{a}}$ & $43.74 \pm 2.78^{\mathrm{a}}$ \\
\hline PU & $29.69 \pm 9.20^{\mathrm{a}}$ & $29.78 \pm 8.94^{\mathrm{a}}$ & $30.94 \pm 6.21^{\mathrm{a}}$ & $29.66 \pm 7.51^{\mathrm{a}}$ & $30.91 \pm 5.29^{\mathrm{a}}$ & $29.73 \pm 4.46^{\mathrm{a}}$ & $29.45 \pm 5.03^{\mathrm{a}}$ \\
\hline U & $71.45 \pm 5.42^{\mathrm{a}}$ & $72.11 \pm 4.72^{\mathrm{a}}$ & $73.49 \pm 3.92^{\mathrm{a}}$ & $74.15 \pm 5.75^{\mathrm{a}}$ & $74.63 \pm 3.93^{\mathrm{a}}$ & $72.77 \pm 2.68^{\mathrm{a}}$ & $73.20 \pm 3.20^{a}$ \\
\hline
\end{tabular}

S: sum of saturated fatty acids; $\mathrm{MU}$ : sum of monounsaturated fatty acids; PU: sum of polyunsaturated fatty acids; U: sum of unsaturated fatty acids.

$a-b$ Means within the same row not followed by the same letter differ significantly $(p<0.05)$.

different sources of the fat used in the manufacture of these sausages. Pigs slaughtered for domestic consumption are usually castrated to avoid the problem of the "boar taint" and, as it is known, this castration diminishes the degree of unsaturation of fat (Njari et al., 1991; Lowe et al., 1992). Also, though dewlap and bacon are fundamentally used in the formulation of the mass of sausages, skin with adhered subcutaneous fat is also used in the artisanal manufacture; this subcutaneous fat, fundamentally dorsal fat, is a hard fat and therefore more saturated.

\subsection{Free fatty acids}

Tables IV and $\mathrm{V}$ show the evolution of the free fatty acids (expressed as $\mathrm{mg} / 100 \mathrm{~g}$ of fat) in the batches of "Chorizo de cebolla" made by artisanal and industrial methods, respectively. Tables VI and VII show the evolution of the free fatty acids expressed as \% of the total free fatty acids. As occurred in the total fatty acid content, high standard deviation values were observed in each sampling point. This variability is due to the different degree of lipolysis observed in the four different batches of each manufacture type. This unequal degree of lipolysis is a consequence of a different activity of the microorganisms and enzymes which operate during ripening.

The total free fatty acids content, expressed as $\mathrm{mg} / 100 \mathrm{~g}$ of fat, underwent throughout ripening a progressive and significant increase $(p<0.05)$ from average values of $459.83 \pm 243.06$ in the mass $(0$ days) to $3687.90 \pm 1670.01$ after 42 days of ripening in the artisanal manufactured sausages, and from $560.34 \pm 317.28$ to $5157.09 \pm 3673.69$ in the industrially manufactured ones. The final values are within the range described in the bibliography for other sausages, the free fatty acids representing between 1 and $7 \%$ of the total fat (Lois et al., 1987; Nagy et al., 1989; Domínguez Fernández and Zumalacárregui Rodríguez, 1991; Montel et al., 1993; Johansson et al., 1994).

Throughout the whole ripening process, significant differences $(p<0.05)$ were not found among the total free fatty acids content, when both types of sausages were compared in each sampling point, owing to the great variability of the data found 
in the different batches corresponding to the same type of manufacture.

However, the evolution followed by the total free fatty acids content in one sausage and in another one was very differentiated. In the artisanal sausages, these increased gradually throughout the ripening process, a increase well defined by a curve of linear regression with a regression coefficient of 0.987 . In the industrial sausages the evolution showed two very defined tendencies: in the first 21 days of ripening the contents increased gradually but less than in the artisanal sausages, a increase well defined by a curve of linear regression with a coefficient of regression of 0.979 ; from thereon, the increase was more pronounced and at the end of ripening the average values found were 1.4 times higher than those found in artisanal sausages.

The rates found for each of the free fatty acids must be the balance between the release by hydrolysis of the glycerides and phospholipids and their oxidative degradation. During ripening of the sausages, peroxides and carbonyls are formed from the free fatty acids released, these oxidative processes fundamentally affect the unsaturated acids and in greater way the polyunsaturated ones (Demeyer et al., 1974). The highest content in free fatty acids showing in the sausages manufactured by industrial methods seems to be owing to the more intense lypoliysis in first place, but the existence of a higher degradation by oxidation of the unsaturated fatty acids in the sausages manufactured by artisanal methods can also be of influence. When compared the percentages of the different free fatty acids in both types of sausages it can be seen that in the industrial sausages the percentage of the unsaturated free fatty acids stays practically constant throughout the ripening process at a value of $73-74 \%$. In the artisanal sausages, this percentage decreases slightly after 21 days of ripening, a decrease which coincides chronologically with the increase in the values of the indexes of peroxides and of the TBA (Salgado et al., 1999). The oxidative phenomena seem to be more important in the artisanal sausages than in the industrial ones, as indicated by the higher indexes of peroxides and of the TBA showed in the artisanal sausages (Salgado et al., 1999). The lower severity of the oxidative phenomena in the industrial sausages could be explained by their higher content in nitrates and nitrites (added deliberately to the mass, see material and methods section) which have a pronounced antioxidant action (Watts, 1954; Cross and Ziegler, 1965). The lower severity of the oxidative processes in the industrial sausages when compared with the artisanal ones has also been shown by other authors (Domínguez Fernández and Zumalacárregui Rodríguez, 1991).

Parallel to the increase of the total free fatty acids, each of the individual free fatty acids also increased throughout the ripening process in both types of sausages. Significant differences $(p<0.05)$ in the arachidonic and palmitoleic acids were found between both types of sausages after 42 days of ripening, with higher contents in the industrial sausages. Though significant differences in the rest of the fatty acids were not found, in general their contents were also higher at the end of ripening in the industrial sausages. The majoritary free fatty acids at the end of ripening were of this order oleic, linoleic and palmitic acids, which sum represented more than $83 \%$ of the total free fatty acids in both types of sausages. This profile of free fatty acids is similar to that found in the bibliography for other sausages (Nagy et al., 1989; Domínguez Fernández and Zumalacárregui Rodríguez, 1991; Montel et al., 1993; Johansson et al., 1994).

Hydrolysis of the lipids has traditionally been attributed above all to the activity of the microbial lipases (Cantoni et al., 1966; Demeyer et al., 1973; Palumbo and Smith, 1977; Nielsen et al., 1989; Papon et al., 1990), though recent studies showed the existence of an intense activity of the lipases of the muscle and the adipose tissue (García et al., 1992; Montel et al., 1993; Molly et al., 1996, 1997; Hierro et al., 1997). According to some studies (Hierro et al., 1997) the lypolytic activity of the tissue lipases could reach $70 \%$ of the total lypolytic activity.

The lipases which act in sausages, as much as those of microbial origin as well as the autochthonous of the tissues, are characterized for the most part by their specificity of action, and preferentially hydrolyze the external positions in the triglyceride molecule, that is, sn1 and sn3 positions (Alford et al., 1971). The triglycerides of pork fat show a characteristic distribution of the fatty acids, in such a way that the greater part of the stearic acid (around $60 \%$ ) is found in the position sn1 of the glycerol, the palmitic acid is esterified in the most part (60-80\%) in the position sn2 and about $50-60 \%$ of the octadecanoic acids (oleic, linoleic and linolenic) are found in the position sn3 (Brockerhoff, 1966).

The results obtained by us agree partially with the information given by these authors. Comparing the percentage values of the different individual fatty acids, when expressed as $\%$ of the total fatty acids and when expressed as \% of the total free fatty acids, it can be observed that the oleic acid was that which was released in more quantities, while the palmitic acid was found among those which was released less intensely.

\section{REFERENCES}

Alford, J.A., Smith. J.L. and Lilly, H.D. (1971). «Relationship of microbial activity to changes in lipids foods». J. Appl. Bacteriol., 34, 133-146.

Antequera, T., García, C., López, C., Ventanas, J., Asensio, M.A. and Córdoba, J.J. (1994). «Evolución de distintos parámetros físico-químicos durante la elaboración de 
jamones ibéricos a partir de cerdos lbéricos (100\%) e lbéricos x Duroc (50\%)». Rev. Agroquím. Tecnol. Aliment., 34(2), 178-200.

Brockerhoff, H. (1966). «Fatty acids distribution patterns of animal depot fats». Compos. Biochem. Physiol., 19, 1-4.

Cantoni, C., Molnar, M.R. and Renon, P. (1966). «Lipasi delle Micrococcaceae e lipolisi del graso suino». Arch. Vet. Ital.., 17, 401-411.

Cava, R., Ruíz, J., López Bote, C., Martín, L., García, C., Ventanas, J. and Antequera, T. (1997). «Influence of finishing diet on fatty acid profiles of intramuscular lipids, triglycerides and phospholipids in muscles of the Iberian pig». Meat Sci., 45, 263-270.

Cross, C.K. and Ziegler, P. (1965). "Comparison of the volatile fractions from cured and uncured meat». $J$. Food Sci., 30, 610-614.

Davenel, A., Riaublanc, A., Marchal, P. and Gandemer, G. (1999). "Quality of pig adipose tissue: relationship between solid fat content and lipid composition». Meat Sci., 51, 73-79.

Demeyer, D., Hoozee, J. and Mesdom, H. (1973). "Specificity of lipolysis during dry sausage ripening». Proc. Eur. Meeting Meat Res. Workers, 19, 1607-1622.

Demeyer, D., Hoozee. J. and Mesdom, H. (1974). «Specificity of lipolysis during dry sausage ripening». J. Food Sci., 39, 293-296.

Domínguez Fernández, M.C. and Zumalacárregui Rodríguez, J.M. (1991). «Lipolytic and oxidative changes in "chorizo" during ripening". Meat Sci., 29, 99-107.

Folch, J., Lees, M. and Stanley, G.H.S. (1957). «A simple method for the insolation and purification of total lipides from animal tissues». J. Biol. Chem., 226, 497-509.

García, M.L., Selgas, M.D., Fernández, M. and Ordóñez, J.A. (1992). "Microorganisms and lipolysis in the ripening of dry fermented sausages». Int. J. Food Sci. Technol., 27, 675-682.

García Fontán, M.C., Vilar, I., Castaño, A., Marra, A.I., Tornadijo, M.E. and Carballo, J. (1998). "Microbiological changes during the ripening of "Chorizo de cebolla", a traditional fermented sausage made in the NW of Spain». Proc. 44th Int. Congress Meat Sci. Technol., II, 838-839.

García Regueiro, J.A. and Díaz, I. (1997). «Considerations on the implementation of triglyceride analysis in determining feeding for the lberian pig». Eurocarne, 7 , 75-79, 101

Hierro, E., de la Hoz, L. and Ordóñez, J.A. (1997). "Contribution of microbial and meta endogenous enzymes to the lipolysis of dry fermented sausages». J. Agric. Food Chem., 45, 2989-2995.

Johansson, G., Berdagué, J.L., Larsson, M., Tran, N. and Borch, E. (1994). «Lipolysis, proteolysis and formation of volatile components during ripening of a fermented sausage with Pedioccocus pentosaceus and Staphylococcus xylosus as starter cultures». Meat Sci., 38, 203-218.

Langner, H.J. (1969). «The formation of free amino acids, volatile fatty acids and volatile carbonyls in dry sausage during maturing». Fleischwirtschaft, 49 , 1475-1479.

Leistner, L. and Dresel, J. (1986). "Chinese raw dry sausage - an alternative technology». Mitteilung. Bundesans. Fleischforsch., 92, 6919-6926.

Leszczynski, D.E., Pikul, J., Easter, R.A., McKeith, F.K., McLaren, D.G., Novakofski, J., Bechtel, P.J. and Jewell, D.E. (1992). «Characterization of lipid in loin and bacon from finishing pigs feed full-fat soybeans or tallow». J. Anim. Sci., 70(7), 2175-2181.
Lois, A.L., Gutiérrez, L.M., Zumalacárregui, J.M. and López, A. (1987). "Changes in several constituents during the ripening of Chorizo - a Spanish dry sausage». Meat Sci., 19, 169-177.

Lowe, D.B., Kempster, A.J., Fogden, M.W. and White, C.F. (1992). «Lipid content and fatty acid profiles of pig meat». Proc. Nutr. Soc., 51(3), 140A.

Lluch, M.C., Pascual, J., Parcerisa, J., Guardiola, F., Codony, R., Rafecas, M. and Boatella, J. (1993). «Trans isomers contents of the fatty acids in meat products. II. Adipose tissue and intramuscular fat from pork». Grasas y Aceites, 44(2), 97-100.

MAPA (1983). "Catálogo de Embutidos y Jamones Curados de España». Secretaría Técnica del Ministerio de Agricultura, Pesca y Alimentación. Madrid.

MAPA (2000). «Alimentación en España: producción, industria, distribución y consumo". Ministerio de Agricultura, Pesca y Alimentación- MERCASA. Madrid.

Molly, K., Demeyer, D., Civera, T. and Verplaetse, A. (1996). «Lipolysis in a Belgian sausage: relative importance of endogenous and bacterial enzymes». Meat Sci., 43, 235-244.

Molly, K., Demeyer, D., Johansson, G., Raemaekers, M., Ghistelinck, M. and Geenen, I. (1997). "The importance of meat enzymes in ripening and flavour generation in dry fermented sausages". Food Chem., 59, 539-545.

Montel, M.C., Talon, R., Berdagué, J.L. and Cantonnet, M. (1993). «Effects of starter cultures on the biochemical characteristics of french dry sausages». Meat Sci., 35, 229-240.

Nagy, A., Mihályi, V. and Incze, K. (1989). «Ripening and storage of Hungarian salami. Chemical and organoleptic changes». Fleischwirtschaft, 69, 587-588.

Nielsen, H.J.S. and Kemmer, M.K.B. (1989). "Lipolitic activity of meat starter cultures». Proc. 35th Int. Congress Meat Sci. Technol., pp. 318-322.

Njari, B., Zivkovic, J., Martincic, T. and Svetina, A. (1991). «Influence of gender on fatty acid composition of pork». Hrana I Ishrana, 32(3), 123-125.

Palanska, O., Hetenyi, L., Ondrejicka, R., Mojto, J. and Kmet'ova, E. (1993). «Fatty acid composition of intramuscular fat of the longissimus lumborum et thoracis in pigs of various slaughter weights". Zivocisna Vyrova, 38(4), 377-384.

Palumbo, S.A. and Smith, J.L. (1977). "Chemical and microbiological changes during sausage fermentation and ripening", in "Enzymes in Food and Beverage Processing». L. Ory and J. St. Angelo (Ed.). ACS Symposium Series, 47, 279.

Papon, M., Talon, R. and Montel, M.C. (1990). «The lipolytic flora of meats and meat products». Viandes et Produits Carnés, 11, 49-55.

Pederson, C.S. (1971). «Microbiology of Food Fermentations». The AVI Publishing Company Inc., Westport (Connecticut).

Pfalzgraf, A., Frigg, M., Steinhart, H., Kirchgessner, M. and Roth, F.X. (1995). "Influence of dietary fat and vitamin E on the lipids in pork meat». Fett Wiss. Technol., 97(1), 13-20.

Rodríguez, M.P., Carballo, J. and López, M. (2001). "Caracterización de la fracción lipídica de algunos productos cárnicos tradicionales gallegos». Grasas y Aceites, 52(5), 291-296.

Roncalés, P., Aguilera, M., Beltrán, J.A., Jaime, I. and Peiró, J.M. (1991). «The effect of natural or artificial casing on the ripening and sensory quality of a mould 
covered dry sausage». Int. J. Food Sci. Technol., 26, 83-89.

Salgado, A., Cruz, J.M., López, M. and Carballo, J. (1999). «Estudio de los procesos lipolíticos y autooxidativos durante la maduración del chorizo de cebolla, un embutido tradicional gallego». Actas XIII Encontro Galego-Portugués de Química. Vigo (Pontevedra, Spain), pp. 237-238.

Santamaría, I., Lizárraga, T., Astiasarán, I. and Bello, J. (1992). "Contribución a la tipificación del chorizo de Pamplona. Estudio físico-químico y sensorial». Rev. Esp. Cienc. Tecnol. Aliment., 32, 431-445.

Scheeder, M.R.L., Glaeser, K.R., Eichenberger, B. and Wenk, C. (2000). «Influence of different fats in pig feed on fatty acid composition of phospholipids and physical meat quality characteristics». Eur. J. Lipid Sci. Technol., 102(6), 391-401.
Shehata, A.J., de Man. J.M. and Alexander, J.C. (1970). «A simple and rapid method for the preparation of methyl esters of fats in milligram amounts for gas chromatography». Canadian Inst. Food Sci. Technol. J., 3, 85-89.

Watts, B.M. (1954). «Oxidative rancidity and discoloration in meats". Adv. Food Res., 5, 1-52.

Wood, J.D., Enser, M., Whittington, F.M., Moncrieff, C.B. and Kempster, A.J. (1989). «Backfat composition in pigs: differences between fat thickness groups and sexes». Livestock Product. Sci., 22(3/4), 351-362.

Recibido: Septiembre 2001 Aceptado: Mayo 2002 